\title{
Prediction of Harmonic Tuning Performance in pHEMTs
}

\author{
Ravi K. Varanasi ${ }^{1}$, Charles P. Baylis II $^{2}$, Lawrence P. Dunleavy ${ }^{2,3}$, William Clausen ${ }^{3}$ \\ ${ }^{1}$ Harris Corporation, Mail stop 102-21C, P.O. Box 37, Melbourne, FL, 32902 \\ ${ }^{2}$ Center for Wireless and Microwave Information Systems, Department of Electrical Engineering, \\ University of South Florida, Tampa, Florida 33620, USA \\ ${ }^{3}$ Modelithics, Inc., 3650 Spectrum Blvd., Suite 170, Tampa, FL 33612
}

\begin{abstract}
This paper focuses on the characterization and optimization of microwave power transistors using a commercial on-wafer harmonic load pull system. Specific attention is paid to the output tuning of the second harmonic impedance presented to the device. The ability to quantify the level of accuracy in a load pull system is explored by using various calibration validation methods. Experiments and simulation comparisons are described for a GaAs pHEMT and a GaAs HJFET. The measured harmonic load pull data pointed to different guidance on how one would match the $2^{\text {nd }}$ harmonic for best performance.
\end{abstract}

\section{INTRODUCTION}

For RF design engineers, who seek to effectively use non-linear simulations, the need to correlate the performance of the power amplifier to a non-linear transistor model is crucial. The PA is the largest source of distortion in a wireless communications system and is extremely challenging to model. The development of a non-linear transistor model is based upon IV characteristics and multi-bias small-signal S-parameters, but a robust model will include characterizations for 50ohm power sweeps and load pull simulations at multiple bias points.

The importance of second-harmonic load pull data has been shown in the literature [e.g. 1]. By use of an optimum secondharmonic termination, for example, the power-added efficiency and output power can be increased. For accurate linearity prediction and design optimization taking fundamental as well as harmonic tuning conditions into account, an accurate large-signal model is necessary.

Of significant interest in the design and optimization of a power amplifier is the performance under varying second harmonic terminations, which can have an impact on the linearity and power capabilities of a device. Much work has been devoted to the enhancement of poweradded efficiency (PAE) [2] via optimization of the second harmonic termination impedance, but the effect of second harmonic tuning on linearity is not well established.

Therefore, for the purposes of this paper, the effects of 2 fo tuning on the linearity figure of merit, Lip3 is explored.
The ability of a model to predict harmonic load tuning results from measurement data will distinguish itself from other models. Once the model can be distinguished in this manner, it can be used to explore harmonic tuning tradeoffs for wider frequency ranges and tuning conditions (i.e. $\Gamma=1)$ than can be realistically achieved on the measurement test bench.

Towards this end, this paper will focus on the characterization and optimization of microwave power transistors utilizing a commercial (Maury Microwave ATS) on-wafer harmonic load pull system, and will provide validation methods to quantify the level of accuracy in the harmonic load pull system. These validation methods include a Delta-Gt calibration routine [3] and the correlation between the small-signal conjugate match (derived from the small-signal S-parameters) and small-signal load pull [4].

\section{BRIEF DISCUSSION OF MODELS}

In this paper, two devices will be examined: a GaAs pHEMT and a GaAs HJFET. A Modelithics extracted EEHEMT model was implemented in Agilent ADS for the pHEMT, while a vendor-supplied TOM scalable model was used for the exploration of the HJFET. For the purposes of this short paper, only data for the pHEMT device will be examined..Also examined for the pHEMT is an Angelov model [7] extracted from both static and pulsed IV measurements.

The EEHEMT model was derived from pulsed IV measurements to form a dynamic model. Multi-bias Sparameters were taken from DC to $30 \mathrm{GHz}$. Gain compression measurements were taken with emphasis on Pout, Gt, PAE, and TOI. Fundamental load pull measurements were then executed using the Maury Microwave load pull system, to form the large-signal model. 


\section{SYSTEM VALIDATION}

To begin the process of validating the accuracy of the harmonic load pull system and of the non-linear EEHEMT model, small-signal S-parameter data for the GaAs pHEMT was taken and compared to the EEHEMT model. Table 1 summarizes the results.

Table 1 Summary of Class AB S-Parameters

\begin{tabular}{|l|l|l|l|l|}
\hline SP Comp. & $\mathrm{S}(1,1)$ & $\mathrm{S}(1,2)$ & $\mathrm{S}(2,1)$ & $\mathrm{S}(2,2)$ \\
\hline Measured & $0.92<-160.3^{\circ}$ & $.03<5.7^{\circ}$ & $5.18<87.4^{\circ}$ & $.496<-161.3^{\circ}$ \\
\hline Modeled & $0.91<-160.2^{\circ}$ & $.03<5.2^{\circ}$ & $5.92<84.4^{\circ}$ & $.445<-156.6^{\circ}$ \\
\hline
\end{tabular}

With the small-signal data indicating reasonably good agreement with the model, the next measurement process was to verify a small-signal conjugate match in the load pull system that was consistent with the measured smallsignal S-parameters. Figure 1 reveals the results for the measurement comparison. This was repeated for the source tuner as well, but data is shown for the load. Table 2 shows the comparison for both source and load. The source correlation is limited by the available tuning range on the measurement test bench. However, the correlation between phases is important as phase loss is very prevalent in the system due to the passive components that comprise the harmonic load pull system.
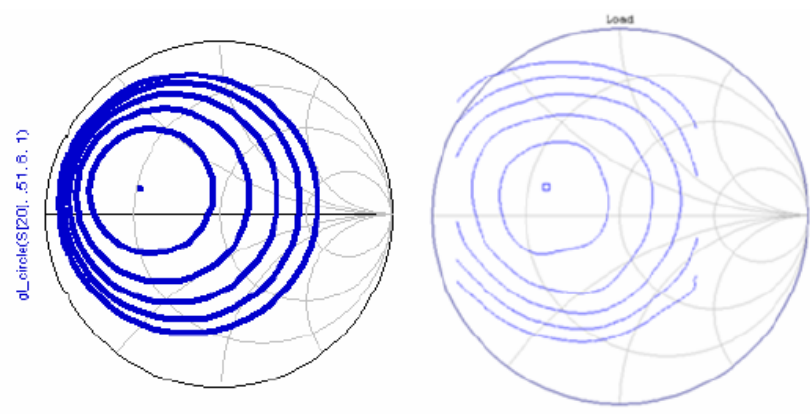

Fig. 1 Small-Signal Load Conjugate Match from Small-Signal S-Parameters (left) and small-signal Load Pull (right)

Table 2 Summary of Class AB Small-Signal Conjugate Match

\begin{tabular}{|l|l|l|}
\hline & Small-Signal S-Param & Small-Signal Tuning \\
\hline Source Conj. Match & $\Gamma=0.92<160.3^{\circ}$ & $\Gamma=0.82<159.46$ \\
\hline Load Conj. Match & $\Gamma=.496<161.3^{\circ}$ & $\Gamma=0.479<160.15$ \\
\hline
\end{tabular}

The final test for the system validation is a Delta-Gt calibration verification, which verifies that the component S-parameters in the system are accurate. The Delta-Gt calibration check is executed for impedances at the center, intermediate and outside of the Smith Chart. Minimum accuracy $<1 \mathrm{~dB}$ is considered acceptable, or can be set by the user. Delta-Gt subtracts the transducer gain from the system S-parameters from the measured transducer gain:

$$
\Delta G_{t}=G_{t}-G_{t}(s)
$$

The results for the Delta-Gt run for the pHEMT are shown in Figure 2.

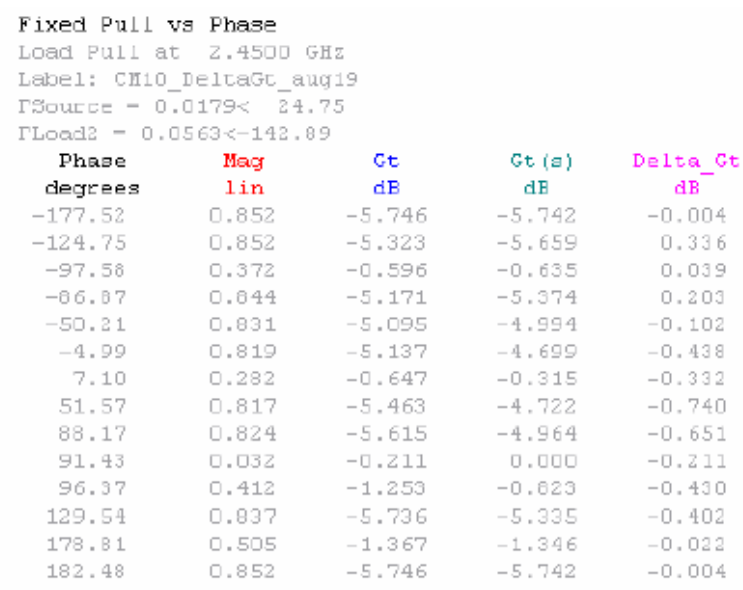

Fig. 2 Delta-Gt Measurement for GaAs pHEMT

\section{FUNDAMENTAL LOAD TUNING FOR L IP3}

The linearity figure of merit, $\mathrm{L}_{\mathrm{ip} 3}$, is a unitless measure that is the ratio of the third-order intercept to the de power:

$$
\operatorname{Lip} 3=\frac{I p 3(m W)}{P d c(m W)}
$$

This figure of merit is used for power amplifiers due to the fact that the third-order intercept relation falls off as the device is driven into compression. Another way to express the performance of the power amplifier/transistor is the IM3 products. With an available input power of $13 \mathrm{dBm}$, measured as the device's $1-\mathrm{dB}$ compression power from a 50 -ohm power sweep (not shown), the fundamental load was optimized for optimum Lip3. The measured and simulated results are shown in Table 3, below. Table 3 shows an excellent correlation between the measured data and EEHEMT model simulation results for fundamental load pull experiments, including termination phases within half a degree of each other. With the fundamental load tuner now optimized and left at this reflection coefficient, the second harmonic was tuned to optimize Lip3. 
Table 3 Summary of Fundamental Load Tuning Results

\begin{tabular}{|l|l|l|l|}
\hline & Pout $(\mathrm{dBm})$ & Lip3 (unitless) & IM3 (dBc) \\
\hline $\begin{array}{l}\text { Simulations } \\
\Gamma=0.486<154.1^{\circ}\end{array}$ & 27.44 & 1.7 & -20.3 \\
\hline $\begin{array}{l}\text { Measurements } \\
\Gamma=0.43<153.9^{\circ}\end{array}$ & 27.27 & 2.2 & -22.42 \\
\hline$|\Delta|$ & 0.17 & 0.5 & 2.12 \\
\hline
\end{tabular}

\section{SECOND HARMONIC LOAD TUNING FOR L $\mathrm{L}_{\text {IP3 }}$}

The second harmonic load impedance is optimized in a different fashion than that of the fundamental load tuner. Instead of varying magnitude and phase, as in the case of the $\mathrm{f}_{\mathrm{o}}$ load tuner, the second harmonic load tuning measurement will present the device with an (ideally) constant magnitude termination of 0.8 , which is the maximum tuning range of the 2 fo tuner. Consequently, the phase of the 2 fo tuner will be varied from 0 to 360 degrees, with the phase increments determined by the characterization of the tuner and the power calibration. This allows us to present the device with either an open circuit $\left(0^{\circ}\right)$ or short circuit $\left(180^{\circ}\right)$ termination. The results for the calculated TOI are shown in Figure 3, with a comparison to the EEHEMT simulated data using the same constant magnitude reflection coefficient of 0.8 . Table 4 summarizes the results between the measurement data and the simulation dataset.

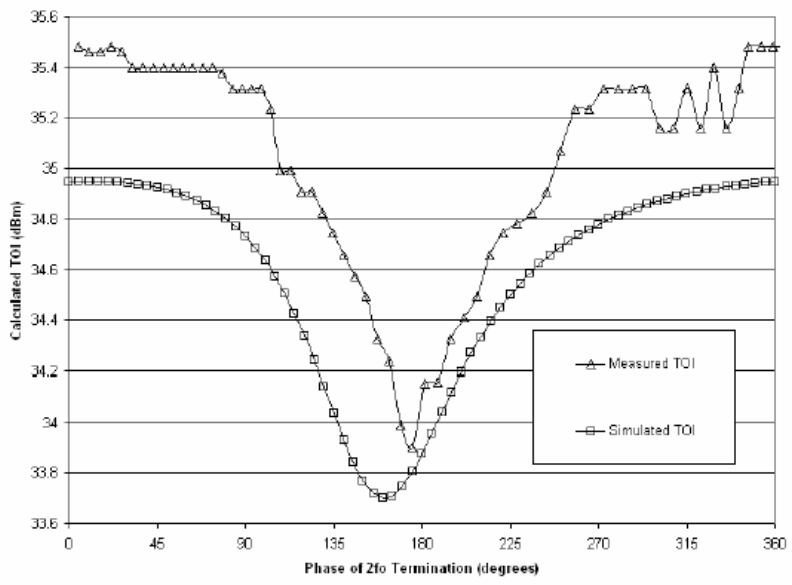

Fig. 3 pHEMT Second Harmonic Load Tuning Comparison @ Pin $=13 \mathrm{dBm}$ and $\Gamma_{\mathrm{L} 2}=0.8$

Table 4 Summary of Second Harmonic Load Tuning Results

\begin{tabular}{|l|l|l|l|}
\hline & Pout $(\mathrm{dBm})$ & Lip3 (unitless) & IM3 $(\mathrm{dBc})$ \\
\hline $\begin{array}{l}\text { Simulations } \\
\Gamma=0.8<10^{\circ}\end{array}$ & 27.53 & 2.09 & 20.867 \\
\hline $\begin{array}{l}\text { Measurements } \\
\Gamma=0.786<4.77^{\circ}\end{array}$ & 27.33 & 2.355 & 23 \\
\hline$|\Delta|$ & 0.2 & 0.265 & 2.13 \\
\hline
\end{tabular}

The measured results are encouraging as there is a similar agreement for Lip3 and IM3 as there was in the fundamental load tuning experiment, and the output power is predicted just as well. These results show that there is minimal improvement in Lip3 that can be achieved using the second harmonic load tuning test bench for this particular constant reflection coefficient magnitude. However, the fact that the model predicted this characteristic shows that the model does provide a good fit for this measurement data.

With this information and a working non-linear model that can predict second harmonic load tuning performance, the EEHEMT model simulations can be used to extend the investigation to foresee what kind of behavior the device would exhibit if we were able to present the device with a maximum constant magnitude of $\Gamma_{\mathrm{L} 2}=1$. Figure 4 shows the variation of the IM3 products as the magnitude of the second harmonic load reflection coefficient is varied from 0.2 to 1.0 in steps of 0.2 and as the phase is simultaneously varied from 0 to 360 degrees. This analysis leads to the observation that the deviation between worst and best case linearity under $2^{\text {nd }}$ harmonic tuning significantly increases as the magnitude of the $2^{\text {nd }}$ harmonic reflection coefficient approaches 1.

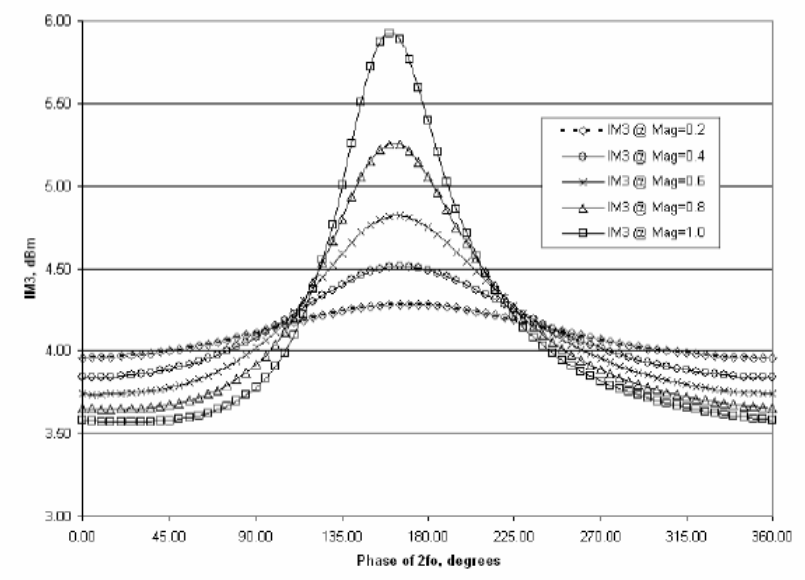

Fig. 4 IM3 (dBm) vs. 2nd Harmonic Simulations for pHEMT

A key point that must be made about this process is that once a non-linear model, such as the EEHEMT shown in this paper, has been validated for harmonic tuning conditions, it can then be used to explore harmonic tuningrelated design trade-offs under a wider range of frequency and tuning conditions than can practically be explored on the measurement test bench due to the system losses. In contrast, the other two device models examined in this work were very far off in terms of $2^{\text {nd }}$ harmonic performance prediction. 


\section{AdVANTAge OF PUlSed IV In HARMONIC TUNING PREDICTION}

It is important that a large-signal model accurately simulate the nonlinear behavior of a device under $\mathrm{RF}$ large-signal conditions. It has been shown in the literature that pulsed IV measurements provide more accurate thermal and trapping conditions to predict the operating characteristics [5],[6]. In this work, Angelov model [7] extractions were performed using the Accent Optical Technologies Dynamic i(V) Analyzer (DiVA) hardware and software for the GaAs PHEMT. using both static and pulsed IV data. The IV curves corresponding to the models are shown in Figure 5. The static IV curves have a lower knee voltage and a higher knee, indicating that for a typical Class A load-line, the static model would predict an output power that is too large.

A load-pull simulation was performed for a quiescent bias of $\mathrm{V}_{\mathrm{DS}}=8 \mathrm{~V}, \mathrm{I}_{\mathrm{D}}=162 \mathrm{~mA}$ and compared to measured load-pull data for this point. Two-tone fundamental and second-harmonic load pull results, shown in Tables 5 and 6, demonstrate that an advantage exists in using the pulsed IV model to predict the nonlinear data. As can be seen, the static model predicts an output power that is too large. In both fundamental and secondharmonic load-pull simulations (results are shown for the point of minimum IM3), the output power, IM3, and transducer gain results are closer to the measured data.

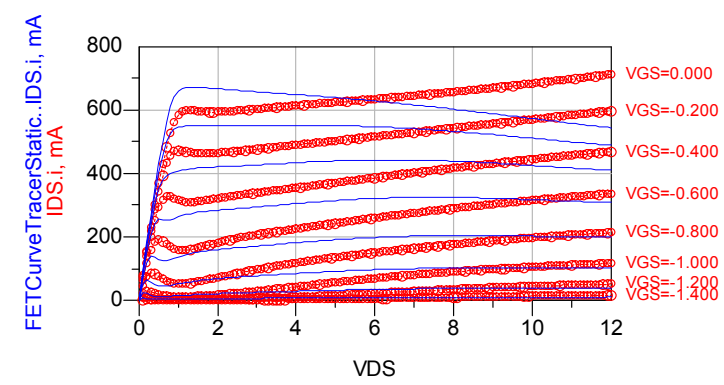

Fig. 5. ADS Plot of Static (Thin Lines) and Pulsed (Thick Lines) IV Simulated Data

TABLE 5: Comparison of Two-Tone Fundamental LoadPull Results

\begin{tabular}{|l|c|c|c|c|}
\hline & $\begin{array}{c}\mathrm{P}_{\text {out }} \\
(\mathrm{dBm})\end{array}$ & $\mathrm{L}_{\mathrm{ip} 3}$ & $\begin{array}{c}\mathrm{IM} 3 \\
(\mathrm{dBc})\end{array}$ & $\begin{array}{c}\mathrm{G}_{\mathrm{T}} \\
(\mathrm{dB})\end{array}$ \\
\hline Meas. & 27.3 & 2.19 & -22.4 & 14.3 \\
\hline Angelov Static & 28.4 & 1.74 & -19.6 & 15.4 \\
\hline Angelov Pulsed & 27.3 & 2.25 & -22.8 & 14.3 \\
\hline
\end{tabular}

TABLE 6: Comparison of Two-Tone Second-Harmonic Load-Pull Results

\begin{tabular}{|l|c|c|c|}
\hline & $\begin{array}{c}\mathrm{P}_{\text {out }} \\
(\mathrm{dBm})\end{array}$ & $\mathrm{L}_{\text {ip3 }}$ & $\begin{array}{c}\text { IM3 } \\
(\mathrm{dBc})\end{array}$ \\
\hline Meas. & 27.3 & 2.36 & -23.0 \\
\hline Angelov Static & 28.5 & 1.82 & -20.0 \\
\hline Angelov Pulsed & 27.5 & 2.36 & -23.1 \\
\hline
\end{tabular}

\section{REFERENCES}

[1] P. Berini, M. Desgagne, F. Ghannevchi, R. Bosisio, "An Experimental Study of the Effects of Harmonic Loading on Microwave MESFET Oscillators and Amplifiers," IEEE Transactions on Microwave Theory and Techniques, Vol. 42, No. 6, June 1994

[2] Rudolph, M., Lenk, F., Doerner, R., Heymann, P., "Influence of $2 \mathrm{GHz}$ Harmonic Load Pull on HBT and MESFET Output Power and Efficiency,"1999 IEEE MTT-S Digest, TUF2-5

[3] Paviol, J., Kueckels, E., Varanasi, R., Dunleavy, L., "PA Load Pull Error Limits using Delta-Gt Contours," UCSD Power Amplifier Conference 2003

[4] Varanasi, R., Liu, J., Paviol, J., Dunleavy, L., Clausen, W., "Using S-Parameter and Load Pull Measurements to Validate Transistor Large-Signal Fundamental and Harmonic Tuning Performance," UCSD Power Amplifier Conference 2004

[5] P. Ladbrooke and J. Bridge, "The Importance of the Current-Voltage Characteristics of FETs, HEMTs, and Bipolar Transistors in Contemporary Circuit Design." Microwave Journal, March 2002.

[6] A. Platzker, A. Palevsky, S. Nash, W. Struble, and Y. Tajima, "Characterization of GaAs Devices by a Versatile Pulsed I-V Measurement System." IEEE Microwave Theory and Techniques Society Digest, 1990.

[7] I. Angelov, H. Zirath, and N. Rorsman, "A New Empirical Nonlinear Model for HEMT and MESFET Devices," IEEE Transactions on Microwave Theory and Techniques, Vol. 40, No. 12, December 1992.

Acknowledgements:

We would like to Conexant for their support of this work and also thank Jim Schellenberg of Schellenberg associates for his past collaborations that led to our studying of measured and modeled second harmonic tuning effects on power transistors. 